\title{
"I Was Scared I Might Die Alone": A Qualitative Study on the Physiological and Psychological Experience of COVID-19 Survivors and the Quality of Care Received at Health Facilities
}

\author{
Isaac Iyinoluwa Olufadewa $a^{1,2^{*}}$, Miracle Ayomikun Adesina ${ }^{1,2}$, Blessing Oladokun ${ }^{2}$, Ararso Baru ${ }^{2,3}$, Ruth \\ Ifeoluwa Oladele ${ }^{1,2}$, Temiloluwa Ololade Iyanda ${ }^{2,4}$, Oluwatosin Joseph Ajibade ${ }^{2}$, Funmilayo Abudu ${ }^{2}$
}

${ }^{1}$ Faculty of Clinical Sciences, College of Medicine, University of Ibadan, Ibadan, Nigeria

${ }^{2}$ Slum and Rural Health Initiative Research Academy, University of Ibadan, Ibadan, Nigeria

${ }^{3}$ College of Medicine and Health Science, Abraminch University, Abraminch, Ethiopia

${ }^{4}$ Department of Public Health, School of Public Health, Southern Medical University, Guangzhou, China

Corresponding Author: Isaac lyinoluwa Olufadewa, MBBS, MHS, Faculty of Clinical Sciences, College of Medicine, University of Ibadan, Ibadan, Nigeria. Tel: +234-8132021189, Email: isaacolufadewa@yahoo.com

Received April 12, 2020; Accepted May 21, 2020; Online Published May 27, 2020

\begin{abstract}
Introduction: Although several coronavirus disease 19 (COVID-19) studies have focused on the biomedical and epidemiological manifestations of the COVID-19 virus, there is a dearth of studies that have reported the experiences of COVID-19 survivors. This study investigated the physiological and psychological experiences of COVID-19 survivors and the quality of care that they received during their recovery processes.

Methods: A phenomenological approach and a purposive sampling technique were employed to select eligible participants whose reported interviews/videos were published on reputable online media channels. The selection processes involved three researchers who had independently searched and assessed the interviews and their sources; for veracity, availability of vital information to meet the study objectives, and to ensure it followed the inclusion criteria. Video interviews were transcribed and data were analyzed using thematic analysis.

Results: Thirty-nine participants (COVID-19 survivors) within the age range of 20-95 from over 15 countries and 5 continents were included in this study. Clinical symptoms commonly reported included feeling feverish, severe, persistent and dry cough, difficulty in breathing, cold, body pains, and aches. Many participants had negative mental health experiences such as being scared, anxious, guilty feelings, and worrying about their recovery. Few participants had positive mental health experiences such as the feeling of encouragement from family and trusted friends. Many participants were satisfied with the quality of care at health centers, though some experienced early difficulty in getting tested.

Conclusion: There are psychological impacts associated with the SARS-CoV-2 infection. Psychological interventions should be included in the management of COVID-19 patients and survivors.

Keywords: COVID-19, Qualitative Study, Mental Health, Physiological Experience, COVID-19 Survivors
\end{abstract}

Citation: Olufadewa II, Adesina MA, Oladokun B, et al. "I was scared i might die alone": a qualitative study on the physiological and psychological experience of COVID-19 survivors and the quality of care received at health facilities. Int J Travel Med Glob Health. 2020;8(2):51-57. doi:10.34172/ ijtmgh.2020.09.

\section{Introduction}

Since the emergence of the novel coronavirus disease 2019 (COVID-19) in December 2019, it has rapidly spread to over 180 countries or territories and as of 6th May 2020 with over 3.7 million cases confirmed globally and over 250000 deaths recorded..$^{1,2}$

COVID-19 has a spectrum of signs and symptoms which varies from asymptomatic or paucisymptomatic forms to clinical conditions such as respiratory failure, that necessitates mechanical ventilation and support in an intensive care unit (ICU), and even systemic multiple organ failure syndromes. ${ }^{3,4}$ Furthermore, it has been discovered that patients with pregnancies, underlying medical conditions, and pre-existing comorbidities may experience a different clinical profile of

Copyright $\odot 2020$ The Author(s). This is an open-access article distributed under the terms of the Creative Commons Attribution License (http:// creativecommons.org/licenses/by/4.0), which permits unrestricted use, distribution, and reproduction in any medium, provided the original work is properly cited. 
the disease when compared to other groups of patients. ${ }^{3,5}$

However, the effects of the COVID-19 pandemic on survivors go beyond physical signs and symptoms - the psychological impact is immediate and can even be longlasting. Behavioral and attitudinal psychological responses of fear, paranoia, and panic, anxiety and experiences of mental health disorders have been recognized in survivors of previous infectious disease outbreaks such as severe acute respiratory syndrome (SARS) and the Middle East respiratory syndrome (MERS) coronaviruses. ${ }^{6,7}$

Conducting a search in 9 databases (PubMed, Google Scholar, Scopus, JURN, CINAHL, Embase, DOAJ, PsycINFO, and Web of Science), we found several COVID-19 studies focusing on the biomedical and epidemiological manifestations of the COVID-19 virus, and a dearth of studies reporting the experiences of COVID-19 survivors who have been treated in a health facility and recovered from the infection. This study aims to investigate the physiological and psychological experiences of COVID-19 survivors during their recovery process which will help to implement a more specific and holistic approach for the short- and longterm care of the patients and survivors of the COVID-19 pandemic. Meanwhile, the gap between our response to emergency healthcare situations and mental health care can be filled through findings from COVID-19 survivors. ${ }^{8}$

\section{Methods}

Study Design and Participants

Our study adopted the phenomenological approach to qualitatively analyze the physiological and psychological experiences of survivors of the SARS-CoV-2 infection who received treatment during their infection in hospitals or isolation centres. The phenomenological approach explores the feelings and experiences of participants and finds shared patterns rather than individual characteristics. ${ }^{9}$ We used this scientific approach to guarantee that the experiences of the research subjects adhere to rigorous scientific standards and to ensure the credibility and reliability of the result. A purposive sampling method was employed to select participants (COVID-19 survivors) who had personal interviews with reputable media agencies that were published online. The selection process involved three researchers (IIO, MAA, and AB) who independently checked-out the published interviews/stories and the sources; for veracity, availability of vital information to meet the study objectives and to ensure it followed the inclusion criteria as follows: (1) the media interview/report must be directly from a COVID-19 survivor (2) the media interview/report must be with a reputable news agency (3) the media interview/reported experience must be verified as an authentic story by all three independent researchers.

\section{Data Collection and Analysis}

Following an independent and extensive search of valid media interviews of COVID-19 survivors over the internet between December 15, 2019 and April 18, 2020, published media interviews/reports of 83 survivors were assessed, but 39 participants were deemed eligible for the study based on the inclusion criteria.

The data employed for this study were sourced, either in online news interview publications or videos (that were later transcribed) from various reputable media platforms including - Washington Post, CNN, AFP, Associated Press, Bloomberg News, Japan Times, Channel News Asia, SKY News, The Canadian Press.

The videos were transcribed verbatim by two researchers (BO and IIO), and a thorough accuracy check was conducted by another researcher (MAA) who listened to the vocals from the videos while reading the transcriptions.

A thematic analysis was carried out at a semantic level, to explore the major themes captioned in each participant's story that fit into the study objectives. The analysis was done such that information accounted by the participants were not attempted to fit into a preexisting coding frame. The coding for the cases and the categorizing of the themes were performed with the aid of the NVIVO-12 software.

\section{Results}

A total of 39 people within the age range of 20-95 across 15 countries from 5 continents (Africa, South America, North America, Europe, and Asia) participated in this qualitative study (Figure 1).

The study enrolled 13 adults (aged 36-64 ), 11 middle-age adults (aged 25-35 ), 6 elderly people (aged 65 or older ) and 4 young adults (aged 20-24). Twenty-one of the participants were females and 18 were males.

The participants included individuals from various occupational backgrounds, including healthcare workers, students, lawyers/attorneys, teachers or school staffs, an analyst, a health and social worker, a church worker, a university professor, a revenue officer, and an executive director at an non-governmental organization.

Three important themes from the analysis of the participants' responses are outlined below:

\section{A. The Physiological Experiences of Participants}

The most commonly mentioned physiological experiences were feeling feverish, severe, persistent and dry cough, difficulty in breathing, cold and chill, pains, and aches in the muscles, chest, and the entire body. Other physiological experiences shared by the COVID-19 survivors but with fewer mentions were loss of appetite, sore-throat and tonsillitis, headache at varying grades, dizziness, changes in voice, insomnia, as well as the loss of smell and taste.

\section{Major Mentions \\ "I suffered from a high fever and pains that tortured every part of my body..." \\ "I kept on coughing... I was scared I might die alone." \\ "...It's like my lungs have sacks of rice around them, so when I take a deep breath, I feel pressure." \\ Minor Mentions \\ "I lost my voice. Sometimes, I couldn't make any sound at all. Sometimes, I sounded like a frog." \\ "I could not sleep at all...It lasted for two days..."}




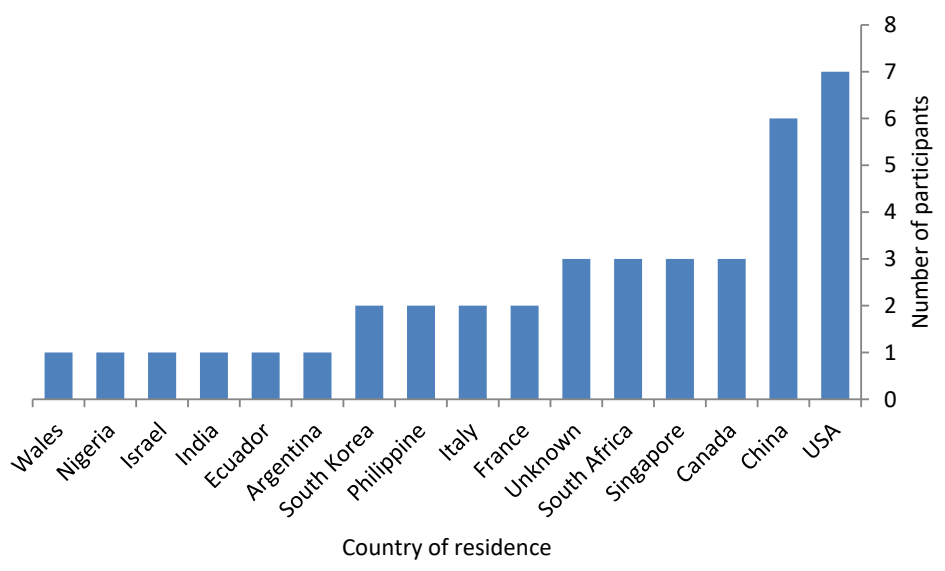

Figure 1. Country of Residence of Study Participants.

\section{B. Mental Health Experiences During Recovery of Participants}

The study found some of the mental health experiences of the participants while recovering from the novel coronavirus (COVID-19). The reported experiences revealed that many of these participants had a negative mental health experience, while few had a positive mental experience (Figure 2). The major sub-themes surrounding the negative mental experiences were being scared, felt panicky and having moments of paranoia; some recalled they had shown anxiety and worry as they tried to recover; one of the participants recalled her feelings of guilt and embarrassment from thoughts of having infected people she came in contact with; some of the participants recounted they were backlashed and treated harshly by people around them, as information about their health status was rumored in their locality.

Stigmatization and misjudgment were also one of the mental health consequences accounted for by the survivors; some reported they had gone through the moment when they cried and shivered over thoughts of having contracted the virus; nightmares and suicidal thoughts were recounted by a few; while some lamented they had to suffer the psychological effect of lacking the usual interpersonal relationships; some expressed their overwhelming and painful memories in the course of their recoveries.

"It was difficult that I was alone on my birthday, I was isolated physically, mentally. A lot of bad thoughts occurred to me...I lost willingness to live. My mental health also took a hit. It was like I lost my goals so I cried."

"I was scared...countless cases were piling up on the desks, and every single doctor was wearing protective clothes, something I'd never seen before..."

"I felt guilty because of the possible risks that my family would be exposed to, both from catching the virus from me and the possibility of being stigmatized by being linked to a confirmed case."

"I faced backlash and harsh comments from the public and even those who know me..."

"I couldn't sleep, anxiety invaded the room...I was afraid of dying without being able to cling on to the hands of my family and friends, despair overcame me."
The study also found some positive experiences among a few of the survivors. One of the participants particularly expressed her journey to recovery that enabled her to learn new things - such as reading books and cooking meals; some reported their relatives and associates that made them feel loved and were encouraged not to get overwhelmed by the illness; a few reported their process of recovery helped them have a better look at life in general, rather than feel down for themselves over life after recovery.

"I thought to myself: It's not my time to die. My husband and I have a 2-year-old son. I want to see him graduate from high school, graduate from college..."

"I was not afraid of the virus as I was hardly showing any symptoms..."

C. Clinical Interventions Needed and Quality of Care During Recovery

Participants reported being administered varying clinical interventions that included but were not limited to intubation, oxygen therapy, heart, and lung bypass among others. They had interventions that were considered appropriate for them at each stage of their disease progression (Figure 3). Some of the participants' responses regarding their clinical interventions are outlined below:

“...was put on heart and lung bypass machines, dialysis, ventilator and feeding tube."

"Oxygen therapy is painful and finding a radial artery is difficult..."

Participants reported that their major challenge was the difficulties they experienced getting tested which could mostly be ascribed to the shortage of test kits. Apart from the difficulties in getting tested, the majority of participants claimed they were satisfied with the quality of treatments they received at various health care centers.

"I can't thank the teams enough for meticulously making sure that my vitals were stable (every two hours) and that I was being administered the right treatment at all times."

However, a few participants were not pleased with how they were treated, especially as regards being updated regularly about their test results.

"I think one thing that can definitely be improved upon is 


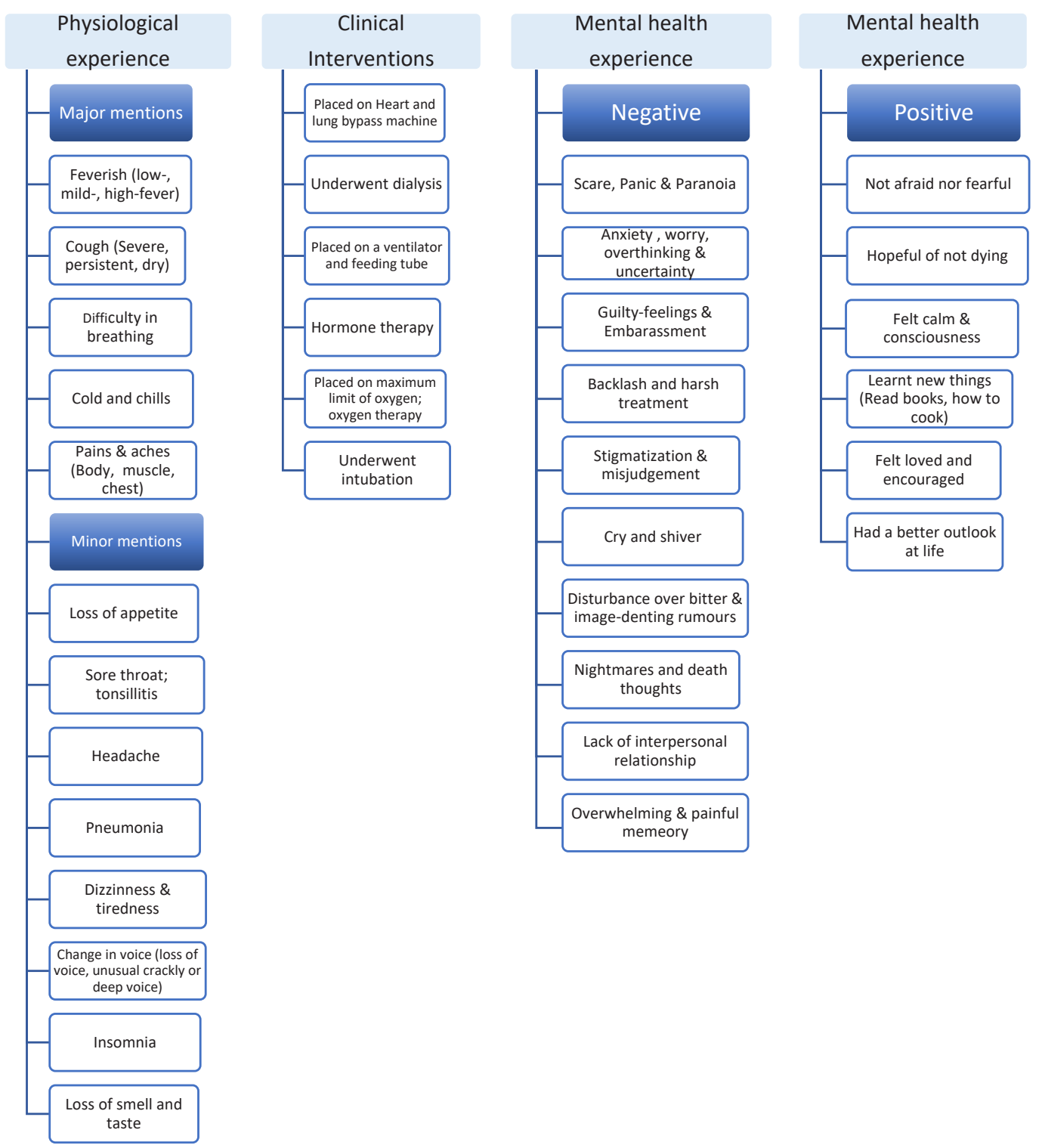

Figure 2. Physiological and Mental Health Experience of Participants and Clinical Interventions Needed During Their Recovery.

communication between the management team and patients because we were kept in the dark about our results..."

\section{Discussion}

This study explored a significant number of physiological and psychological experiences of COVID-19 survivors and the quality of care received at healthcare facilities or isolation centers during their recovery process.

Fever, breathing difficulties, headaches, insomnia, loss of appetite, and pneumonia were among the symptoms reported by our respondents. These physiological experiences are consistent with the data on clinical manifestations of the SARS-CoV-2 infection. ${ }^{10-13}$ The severity and type of symptoms experienced by survivors may differ owing to several reasons, ${ }^{14,15}$ which explains why treatment plans need to be individualized. Some of the COVID-19 survivors were intubated, underwent oxygen therapy, had a heart and lung bypass done amidst others. These clinical interventions meted out are in line with the findings from studies conducted on features, evaluation and treatment of coronavirus. ${ }^{4}$

Participants reported psychological experiences which according to many of them was worse than the physiological or clinical symptoms experienced. This study found that anxiety, panic, fear, and risk perception of death during their recovery were common mental health experiences among many of the COVID-19 survivors. This could be due to the uncertainty about the disease, the isolation experienced by many of them, the situation of COVID-19 treatment center (especially observing health professionals donning full personal protective equipment), and several disturbing viral health misinformation that participants might have been exposed to. These negative feelings experienced by survivors might not only be relevant to the life-threatening nature of the virus but also to the deep concern about not becoming an agent of infecting others. Some participants also experienced worries and guilty feelings which could be due to being bothered that their family might be infected with the deadly virus due to them. Evidence provided by Cheng et al and other 


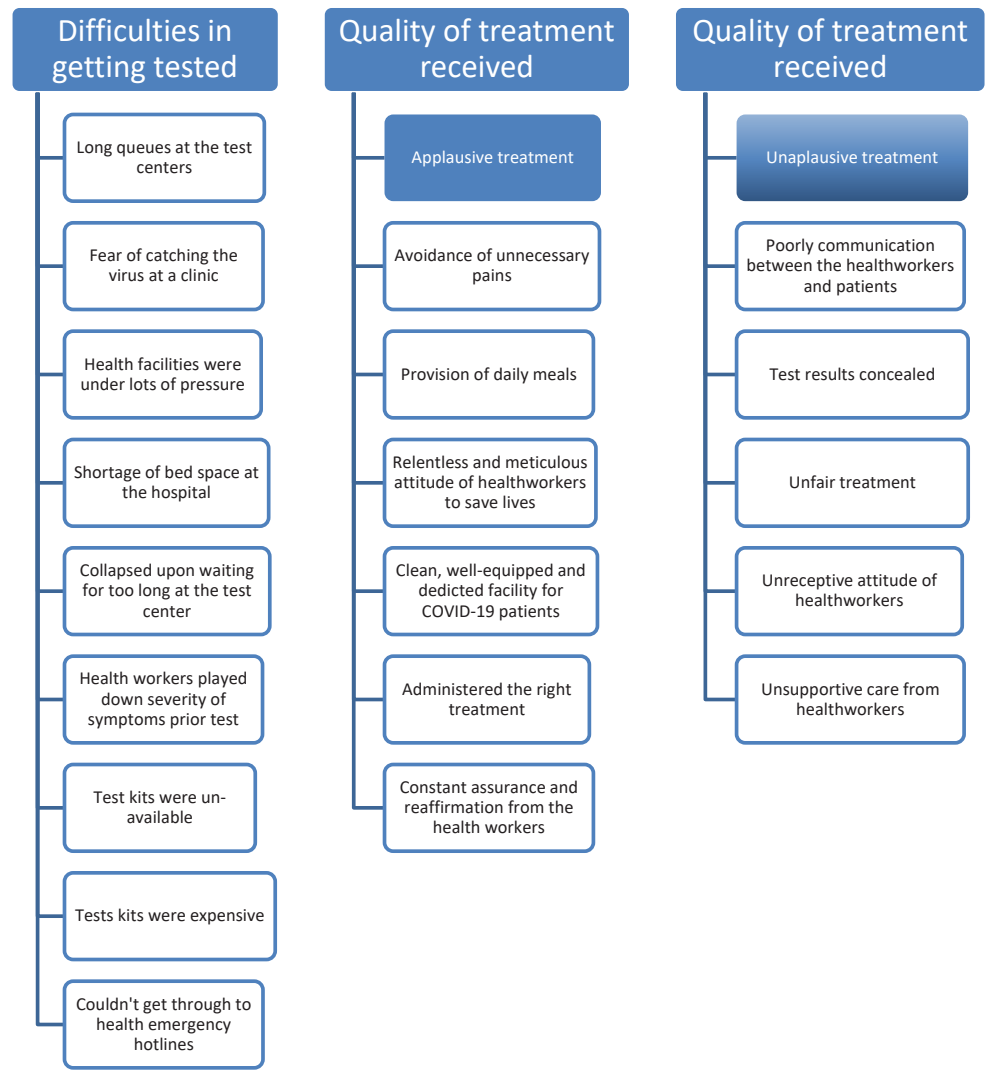

Figure 3. Quality of Treatment Received by Participants.

studies that detail some SARS survivors' negative mental health experiences further corroborate our findings. 716,17 Therefore, reducing risk perception through psychological support and clear communication will be helpful for the mental health wellbeing of COVID-19 patients. Health care professionals and officers must be prepared to provide appropriate emotional supports for COVID-19 patients and survivors to mitigate the associated impact on their mental health.

It is very common for quarantined or isolated people to experience acute mental disorders while recovering from an infection or from a life-threatening incident. ${ }^{18-21}$ Even though it is not clear whether it is due to quarantine or underling pathology, a significant proportion of COVID-19 survivors in this study reported experiences of depression, overwhelming and painful memory while recovering from COVID-19 infection. Isolation and quarantine hinder family support which may worsen the negative psychological experiences of COVID-19 survivors if not timely managed. ${ }^{19,22}$ Hawryluck et al also found similar evidence to support the negative impact of quarantine on patients during the SARS disease outbreak. ${ }^{23}$ However, we do not suggest that isolation or quarantine should not be used but should be as tolerable as possible for the isolated patients which can be achieved through psychological support, clear communication, compassionate healthcare delivery and allowing isolated or quarantined people to use technologies such as social media and telecommunication with their families. ${ }^{24}$
Even though it is too early to know if people who have survived the SARS-CoV-2 infection will experience posttraumatic stress disorder (PTSD), studies conducted among survivors of SARS-CoV and MERS-CoV reported longterm psychiatric conditions such as PTSD and depressive disorders. ${ }^{17,20,21,23}$ The long-term consequences of COVID-19 illness on survivors may be similar to that caused by SARS and MERS. Without early intervention, mental health symptoms experienced by COVID-19 survivors during the recovery process may evolve into long-term PTSD. Thus, symptoms of anxiety, depression, paranoia, guilty feelings, anger, and other mental health-related clinical manifestations should be recognized early and appropriate intervention needs to be implemented for improvement in symptoms to improve the mental health resilience of the COVID-19 survivors.

Studies have shown that positive emotions and optimism play an important role in recovery and reducing psychological trauma. It also helps to promote the psychological rehabilitation of post-traumatic stress disorders. ${ }^{25,26}$ COVID-19 survivors in our study also believed that an infected person should be calm, optimistic, and altruist (by staying in the hospital not in their house with the family) as a psychological adjustment mechanism to the stressors. Therefore, actively stimulating positive emotions and coping styles are crucial to promote the mental health of COVID-19 patients.

Emerging viral pandemics can place extraordinary and sustained demands on public health, health systems, and on providers of essential community services. ${ }^{27,28}$ Survivors of 
COVID-19 in this study experienced numerous challenges in getting tested related to the shortage of test kits and long queues at the test center. Lack of access to testing and negligence may create a favorable environment for the spread of the virus to others. Therefore, health authorities and policymakers must do all they can to prevent the scarcity of medical resources. They should also quarantine the suspected cases instead of sending them back home to prevent community transmission.

Even though many COVID-19 survivors reported encouraging statements regarding the quality of the health care and respect received from health care professionals, some of the respondents had unpleasant experiences and perceived stigmatization. This finding also resonates with experiences of patients from the previous epidemic such as human immunodeficiency virus (HIV), Ebola, MERS-CoV and SARS-CoV..$^{21,29-31}$ Stigma may lead to reluctance to getting tested reducing the confidence to seek help and medical care. Several studies have shown that the stigmatized group may be also subjected to social avoidance, physical violence, denials of housing, and healthcare. ${ }^{21,29,31}$ Hence, there is compelling evidence that proves stigma reduction intervention will not only benefit the mental health of COVID-19 patients but also may help to halt the spread of the virus by encouraging people to seek health care.

\section{Limitations}

Our study involved the analysis of media interviews granted by COVID-19 survivors with reputable media agencies and there is a possibility that there have been errors made during the online publication which might have affected our results. Meanwhile, we do not know if there has been a background history of mental health illness in some of our participants before they got infected with the COVID-19 virus which might have led to a more pronounced negative mental health experience.

\section{Conclusion}

The coronavirus pandemic is presently ravaging our world with millions of cases and hundreds of thousands of deaths have been recorded. Most studies have focused on the biomedical characteristics and epidemiological patterns of the COVID-19 virus; however, there is still a lot unknown about this novel coronavirus especially the psychological experience of survivors. Our study employed a rigorous approach to unearth the mental health experience of coronavirus patients and survivors. We found that the psychological experience of many COVID-19 survivors during the period of recovery was more pronounced than their physiological experience. Our findings would enable utilization of a more comprehensive healthcare strategy, improve mental health care during the clinical management of COVID-19 patients and inform public health policy to ensure that stigma and discrimination of COVID-19 survivors are urgently addressed.

\section{Recommendations}

We recommend psychological support be provided for COVID-19 patients during treatment as this will improve

\section{Research Highlights}

What Is Already Known?

Globally, the focus of most studies has been on the biomedical and epidemiological details of the novel coronavirus and little attention has been paid to the psychological experiences of COVID-19 survivors as evidenced by the dearth of studies found in this area.

\section{What This Study Adds?}

This study presents important findings about the physiological and psychological experiences of COVID-19 survivors. Some of the physiological findings of the novel coronavirus presented are headaches, dizziness, change in voice, cold, and chills. The novel psychological findings presented here include a feeling of guilt about the possibility of having infected the people they have come in contact with, nightmares, suicidal ideation, stigmatization, misjudgment, discrimination among others.

their mental health and response to treatment. Using creative solutions such as attaching their smartphone to a surface for SARS-CoV-2 patients to speak to trusted friends and family on Skype, Zoom or any other video conferencing Apps while on treatment will be helpful. ${ }^{24}$

There is also a need to provide psychological support to COVID-19 survivors after hospital discharge. Psychological interventions could be individualized based on the severity of disease earlier experienced and in cases of background mental health disorders.

Health education on the severity, myths, and facts of the novel coronavirus infection should be given to the public to reduce stigmatization and discrimination associated with receiving recovered patients back into the community.

We recommend the training of healthcare professionals to help them handle the stress at work and provide better clinical psychological care for COVID-19 patients and critically ill patients.

\section{Authors' Contributions}

IIO, MAA, and BO conceived the main concepts of this study. IIO was involved in the data collection, study design, literature review, he was also involved with some parts of the data analysis and wrote the first draft and edited the final draft of the manuscript. MAA was involved in the data collection, literature review, and edited the second draft of the manuscript. BO was involved mainly with data collection, did most of the data analysis, was involved in the literature review, and wrote parts of the first draft of the manuscript. $\mathrm{AB}$ contributed to the literature search, and was involved with data collection, and wrote a part of the second and third drafts. RIO, OJA, and TOI contributed to the literature search and wrote a part of the second and third drafts. FA contributed to the literature search and wrote the final draft of the manuscript. All authors approved the final manuscript. 


\section{Ethical Approval}

Privacy and confidentiality of the participants were maintained, as the names of interviewees and their home addresses are not included in this study.

The Standards for Reporting Qualitative Research (SRQR) guideline was followed in reporting this study. ${ }^{32}$

\section{Conflict of Interest Disclosures}

The authors declare that they have no conflict of interests.

\section{Funding/Support}

The authors did not receive any funding for this work. This was a self-funded study.

\section{References}

1. World Health Organization (WHO). Coronavirus Disease 2019 (COVID-19) Situation Report-104. WHO; 2020. https://www.who. int/docs/default-source/coronaviruse/situation-reports/20200503covid-19-sitrep-104.pdf?sfvrsn=53328f46_2. Accessed May 6, 2020

2. Worldometer. COVID-19 Coronavirus Pandemic. Worldometer website. 2020. https://www.worldometers.info/coronavirus. Accessed May 6, 2020

3. Adhikari SP, Meng S, Wu YJ, et al. Epidemiology, causes, clinical manifestation and diagnosis, prevention and control of coronavirus disease (COVID-19) during the early outbreak period: a scoping review. Infect Dis Poverty. 2020;9(1):29. doi:10.1186/s40249-02000646-x.

4. Cascella M, Rajnik M, Cuomo A, Dulebohn SC, Di Napoli R. Features, Evaluation and Treatment Coronavirus (COVID-19). Treasure Island, FL: StatPearls Publishing; 2020.

5. Chen $\mathrm{H}$, Guo J, Wang $\mathrm{C}$, et al. Clinical characteristics and intrauterine vertical transmission potential of COVID-19 infection in nine pregnant women: a retrospective review of medical records. Lancet. 2020;395(10226):809-815. doi:10.1016/s01406736(20)30360-3.

6. Lee AM, Wong JG, McAlonan GM, et al. Stress and psychological distress among SARS survivors 1 year after the outbreak. Can J Psychiatry. 2007;52(4):233-240. doi:10.1177/070674370705200 405.

7. Cheng SK, Wong CW, Tsang J, Wong KC. Psychological distress and negative appraisals in survivors of severe acute respiratory syndrome (SARS). Psychol Med. 2004;34(7):1187-1195. doi:10.1017/s0033291704002272.

8. Lima CKT, Carvalho PMM, Lima I, et al. The emotional impact of coronavirus 2019-nCoV (new coronavirus disease). Psychiatry Res. 2020;287:112915. doi:10.1016/j.psychres.2020.112915.

9. Colaizzi PF. Phenomenological analysis. In: Polit DF, Beck CT, eds. Essentials of Nursing Research: Methods, Appraisal, and Utilization. Philadelphia: Lippincott Williams \& Wilkins; 1978. p. 409-411.

10. Huang C, Wang Y, Li X, et al. Clinical features of patients infected with 2019 novel coronavirus in Wuhan, China. Lancet. 2020;395(10223):497-506. doi: 10.1016/S0140-6736(20)301835

11. Ren LL, Wang YM, Wu ZQ, et al. Identification of a novel coronavirus causing severe pneumonia in human: a descriptive study. Chin Med J (Engl). 2020;133(9):1015-1024. doi:10.1097/ cm9.0000000000000722.

12. Carlos WG, Dela Cruz CS, Cao B, Pasnick S, Jamil S. Novel Wuhan (2019-nCoV) coronavirus. Am J Respir Crit Care Med. 2020;201(4):P7-P8. doi:10.1164/rccm.2014P7.

13. Wu C, Chen $X$, Cai $Y$, et al. Risk factors associated with acute respiratory distress syndrome and death in patients with coronavirus disease 2019 pneumonia in Wuhan, China. JAMA Intern Med. 2020. doi:10.1001/jamainternmed.2020.0994.

14. Wang W, Tang J, Wei F. Updated understanding of the outbreak of 2019 novel coronavirus (2019-nCoV) in Wuhan, China. J Med
Virol. 2020;92(4):441-447. doi:10.1002/jmv.25689.

15. Rothan HA, Byrareddy SN. The epidemiology and pathogenesis of coronavirus disease (COVID-19) outbreak. J Autoimmun. 2020;109:102433. doi:10.1016/j.jaut.2020.102433.

16. Cheng SK, Tsang JS, Ku KH, Wong CW, Ng YK. Psychiatric complications in patients with severe acute respiratory syndrome (SARS) during the acute treatment phase: a series of 10 cases. $\mathrm{Br}$ J Psychiatry. 2004;184:359-360. doi:10.1192/bjp.184.4.359.

17. Mak IW, Chu CM, Pan PC, Yiu MG, Chan VL. Long-term psychiatric morbidities among SARS survivors. Gen Hosp Psychiatry. 2009;31(4):318-326. doi:10.1016/j.genhosppsych.2009.03.001.

18. Ellepola A, Hospital AT. Foreseeable psychological impact of COVID 19 in Sri Lanka. https://www.researchgate.net/profile/ Anuradha_Ellepola2/publication/340522703_Foreseeable_ psychological_impact_of_COVID_19_in_Sri_Lanka/ links/5e8e8e274585150839cac845/Foreseeable-psychologicalimpact-of-COVID-19-in-Sri-Lanka.pdf. Accessed May 2, 2020. Published April 2020.

19. Brooks SK, Webster RK, Smith LE, et al. The psychological impact of quarantine and how to reduce it: rapid review of the evidence. Lancet. 2020;395(10227):912-920. doi:10.1016/s01406736(20)30460-8

20. Jeong H, Yim HW, Song YJ, et al. Mental health status of people isolated due to Middle East Respiratory Syndrome. Epidemiol Health. 2016;38:e2016048. doi:10.4178/epih.e2016048.

21. Almutairi AF, Adlan AA, Balkhy HH, Abbas OA, Clark AM. "It feels like I'm the dirtiest person in the world.": exploring the experiences of healthcare providers who survived MERS-CoV in Saudi Arabia. J Infect Public Health. 2018;11(2):187-191. doi:10.1016/j. jiph.2017.06.011.

22. Hollander JE, Carr BG. Virtually perfect? Telemedicine for Covid-19. N Engl J Med. 2020;382(18):1679-1681. doi:10.1056/ NEJMp2003539.

23. Hawryluck L, Gold WL, Robinson S, Pogorski S, Galea S, Styra R. SARS control and psychological effects of quarantine, Toronto, Canada. Emerg Infect Dis. 2004;10(7):1206-1212. doi:10.3201/ eid1007.030703.

24. Wakam GK, Montgomery JR, Biesterveld BE, Brown CS. Not dying alone - modern compassionate care in the Covid-19 pandemic. $\mathrm{N}$ Engl J Med. 2020. doi:10.1056/NEJMp2007781.

25. Waugh CE. The regulatory power of positive emotions in stress: a temporal-functional approach. In: Kent M, Davis MC, Reich JW, eds. The Resilience Handbook: Approaches to Stress and Trauma. Routledge/Taylor \& Francis Group; 2013. p. 73-85.

26. Carbone EG, Echols ET. Effects of optimism on recovery and mental health after a tornado outbreak. Psychol Health. 2017;32(5):530548. doi:10.1080/08870446.2017.1283039.

27. U.S. Department of Health and Human Services (HHS). Pandemic Influenza Plan - 2017 Update. https://www.cdc.gov/flu/pandemicresources/pdf/pan-flu-report-2017v2.pdf. Accessed May 3, 2020. Published 2017

28. Emanuel EJ, Persad G, Upshur R, et al. Fair allocation of scarce medical resources in the time of Covid-19. N Engl J Med. 2020;382(21):2049-2055. doi:10.1056/NEJMsb2005114.

29. James PB, Wardle J, Steel A, Adams J. An assessment of Ebolarelated stigma and its association with informal healthcare utilisation among Ebola survivors in Sierra Leone: a cross-sectional study. BMC Public Health. 2020;20(1):182. doi:10.1186/s12889020-8279-7.

30. Siu JY. Influence of social experiences in shaping perceptions of the Ebola virus among African residents of Hong Kong during the 2014 outbreak: a qualitative study. Int J Equity Health. 2015;14:88. doi:10.1186/s12939-015-0223-6.

31. Stutterheim SE, Pryor JB, Bos AE, Hoogendijk R, Muris P, Schaalma HP. HIV-related stigma and psychological distress: the harmful effects of specific stigma manifestations in various social settings. AIDS. 2009;23(17):2353-2357. doi:10.1097/QAD.0b013e3283320dce.

32. O'Brien BC, Harris IB, Beckman TJ, Reed DA, Cook DA Standards for reporting qualitative research: a synthesis of recommendations. Acad Med. 2014;89(9):1245-1251. doi:10.1097/ acm.0000000000000388. 